\title{
Operating single quantum emitters with a compact Stirling cryocooler
}

Cite as: Rev. Sci. Instrum. 86, 013113 (2015); https://doi.org/10.1063/1.4906548

Submitted: 22 October 2014 . Accepted: 14 January 2015 . Published Online: 29 January 2015

A. Schlehahn, L. Krüger, M. Gschrey, J.-H. Schulze, S. Rodt, A. Strittmatter (D), T. Heindel, and S. Reitzenstein (D)
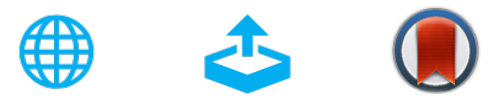

View Online

Export Citation

\section{ARTICLES YOU MAY BE INTERESTED IN}

On-demand generation of background-free single photons from a solid-state source Applied Physics Letters 112, 093106 (2018); https://doi.org/10.1063/1.5020038

Single-photon emission at a rate of $143 \mathrm{MHz}$ from a deterministic quantum-dot microlens triggered by a mode-locked vertical-external-cavity surface-emitting laser Applied Physics Letters 107, 041105 (2015); https://doi.org/10.1063/1.4927429

Invited Review Article: Single-photon sources and detectors

Review of Scientific Instruments 82, 071101 (2011); https://doi.org/10.1063/1.3610677

\section{Lock-in Amplifiers up to $600 \mathrm{MHz}$}
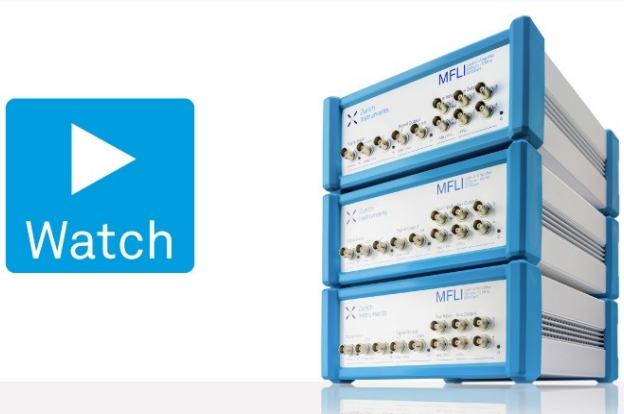


\title{
Operating single quantum emitters with a compact Stirling cryocooler
}

\author{
A. Schlehahn, L. Krüger, M. Gschrey, J.-H. Schulze, S. Rodt, A. Strittmatter, ${ }^{\text {a) }}$ T. Heindel, ${ }^{\text {b) }}$ \\ and S. Reitzenstein \\ Institute of Solid State Physics, Technische Universität Berlin, 10623 Berlin, Germany
}

(Received 22 October 2014; accepted 14 January 2015; published online 29 January 2015)

\begin{abstract}
The development of an easy-to-operate light source emitting single photons has become a major driving force in the emerging field of quantum information technology. Here, we report on the application of a compact and user-friendly Stirling cryocooler in the field of nanophotonics. The Stirling cryocooler is used to operate a single quantum emitter constituted of a semiconductor quantum dot (QD) at a base temperature below $30 \mathrm{~K}$. Proper vibration decoupling of the cryocooler and its surrounding enables free-space micro-photoluminescence spectroscopy to identify and analyze different charge-carrier states within a single quantum dot. As an exemplary application in quantum optics, we perform a Hanbury-Brown and Twiss experiment demonstrating a strong suppression of multi-photon emission events with $\mathrm{g}^{(2)}(0)<0.04$ from this Stirling-cooled single quantum emitter under continuous wave excitation. Comparative experiments performed on the same quantum dot in a liquid helium (LHe)-flow cryostat show almost identical values of $g^{(2)}(0)$ for both configurations at a given temperature. The results of this proof of principle experiment demonstrate that low-vibration Stirling cryocoolers that have so far been considered exotic to the field of nanophotonics are an attractive alternative to expensive closed-cycle cryostats or LHe-flow cryostats, which could pave the way for the development of high-quality table-top non-classical light sources. (C) 2015 AIP Publishing LLC. [http://dx.doi.org/10.1063/1.4906548]
\end{abstract}

\section{INTRODUCTION}

The emerging field of quantum information technology has become a major driving force for the development of easy-to-operate single-photon sources (SPSs) $)^{1-5}$ and detectors. ${ }^{3,6}$ To realize practical solid state based SPSs, mainly two different routes can be pursued: first, specific materials can be used to achieve room temperature operation, and second, an easy-to-handle cooling technique can be applied to operate readily available high-quality single-photon emitters at cryogenic temperatures. Promising candidates for the first approach are, e.g., nitrogen vacancy centers in diamond nanocrystals. ${ }^{7,8}$ However, at room temperature, these defect centers suffer from large emission linewidths and are incompatible with standard semiconductor device technology. Alternatively, semiconductor quantum dots (QDs) based on widebandgap materials have proven their potential to act as singlephoton emitters up to room temperature which is facilitated by their large excitonic binding energies and the tight carrier confinement. ${ }^{9,10}$ Yet, also in this case, the inevitable phonon interaction with the surrounding semiconductor matrix leads to significant broadening of emission lines and to a significant spectral overlap of emission from different excitonic complexes, potentially leading to reduced single-photon purity. ${ }^{11}$ Thus, even though exciting progress has been achieved with respect to the development of quantum light sources operating at room temperature, ${ }^{12}$ the phonon related line broadening and

\footnotetext{
a)Present address: Abteilung Halbleiterepitaxie, Otto-von-Guericke Universität, 39106 Magdeburg, Germany.

b) Author to whom correspondence should be addressed. Electronic mail: tobias.heindel@tu-berlin.de
}

increasing contributions of dephasing will probably hinder their application as high quality single-photon emitters in the context of quantum information technology. In contrast, selforganized Stranski-Krastanov QDs in the $\operatorname{In}(\mathrm{Ga})$ As material system feature close to ideal quantum efficiency, long dephasing times, and narrow emission linewidths, ${ }^{13,14}$ leading to excellent quantum optical properties in terms of singlephoton purity and photon indistinguishability, ${ }^{15}$ in particular, under strict resonant excitation. ${ }^{16-18}$ However, these superb emission features require cryogenic temperatures below about $50 \mathrm{~K}$ - conditions which so far have been realized utilizing either laborious liquid Helium flow cryostats or expensive and power consuming closed-cycle pulse tube systems.

In this work, we pursue an alternative approach to provide high quality single-photons that avoids dealing with fundamental physical limitations at higher temperatures and at the same time eliminates the need for costly liquid helium ( $\mathrm{LHe}$ ) infrastructures or more complex cooling systems, such as closed cycle pulse-tube coolers. We take advantage of the high optical quality of self-assembled InGaAs QDs up to temperatures of a few $10 \mathrm{~K}$ and the availability of compact and low vibration Stirling cryocoolers with a base temperature below $30 \mathrm{~K}$. Up till now, this type of cryocooler has been utilized and optimized particularly for infrared cameras and for applications in space missions, where independence of consumables is crucial. Within the nanophotonics community, Stirling cryocoolers were deemed as "exotic" devices with a base temperature being much too high (according to its specifications typically $>60 \mathrm{~K}$ ) and mechanical vibrations much too large to be considered applicable in free-space optical laboratory experiments. To demonstrate the high potential of this concept for table-top quantum light sources and their application in 
quantum optical experiments, we show Stirling-cooled operation and single-photon emission of single optically pumped QDs deterministically integrated into sub-micrometer mesa structures. Corresponding spectroscopic results are compared to those obtained for the same QD in a "standard configuration" using a LHe-flow cryostat. Since outstanding emission properties are inevitable for a realistic use of SPSs and the physical constraints on room-temperature operation seem not yet negotiable, our approach paves the way for single-photons conveniently available within a practical and user-friendly concept. Similar progress has been reported with respect to the integration of superconducting single-photon detectors into commercially available cryocoolers. ${ }^{19}$

\section{SAMPLE AND SETUP}

To demonstrate the applicability of a commercially available Stirling-cryocooler (RICOR K535LV) ${ }^{20}$ with a base temperature below $30 \mathrm{~K}$ and a cool-down time below half an hour (starting at room-temperature) for the on-demand generation of single-photons from single semiconductor QDs, we integrated the cooler into a free-space micro-photoluminescence $(\mu \mathrm{PL})$ setup for quantum-optical experiments (cf. Fig. 1).
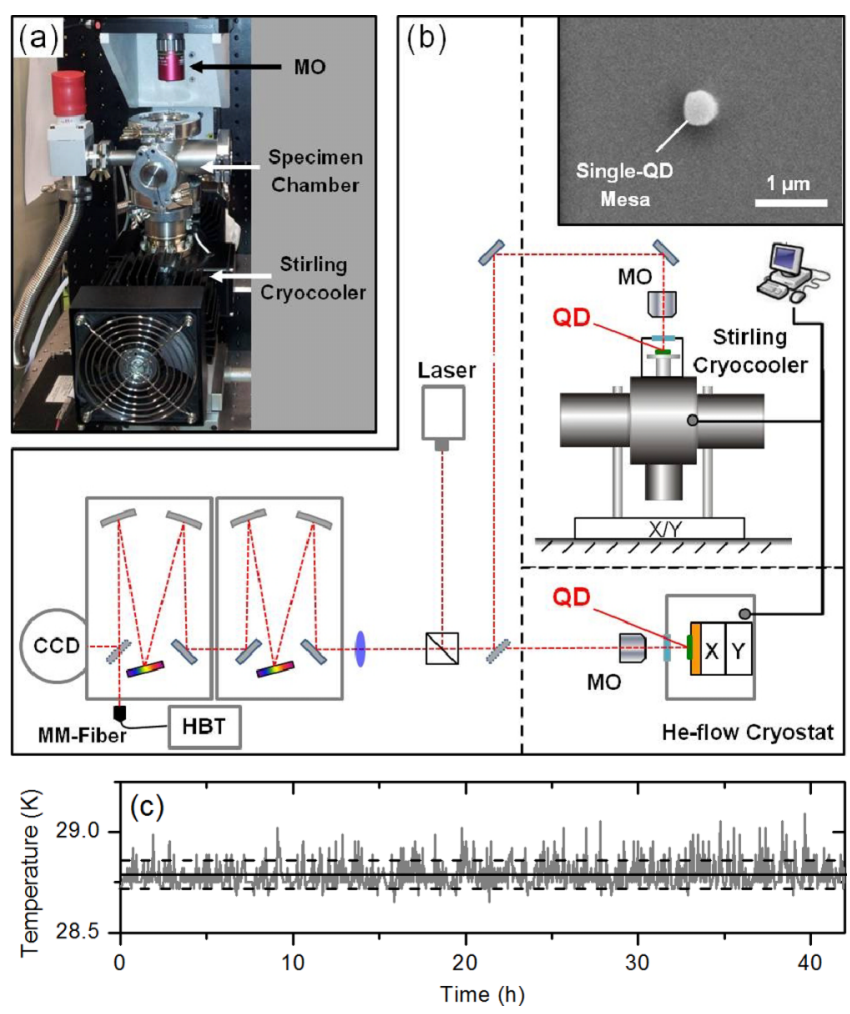

FIG. 1. Measurement setup used for the comparison of conventional LHeflow cooling and Stirling cycle cooling for quantum-optical experiments. (a) Image of the Stirling cryocooler and the microscope objective (MO). (b) Schematic view of the experimental setup, including the mentioned cryostats, an excitation laser, a double monochromator with CCD, and a multi-mode (MM) fiber coupled Hanbury-Brown and Twiss (HBT) module. Inset: Scanning electron microscopy image (top view) of a deterministic single-QD mesa structure with a diameter of $400 \mathrm{~nm}$. (c) Temperature of the Stirling cryocooler's cold-tip during a long-term measurement: the mean temperature is $28.79 \mathrm{~K}$ (full line) and standard deviation is $0.07 \mathrm{~K}$ (indicated by dashed lines).
The Stirling cryocooler was mounted on the same optical table using passive vibration decoupling. For optical excitation and detection of the sample, the cryocooler was equipped with a vacuum specimen chamber. The optical experiments were performed on self-assembled InGaAs QDs (density $\sim 1 \times 10^{9} \mathrm{~cm}^{-2}$ ) grown by metal-organic chemical vapor deposition (MOCVD) in the Stranski-Krastanov growth mode. To enhance temperature stability, the QD layer is sandwiched between two AlGaAs diffusion barriers ( 30 nm) after a 150 $\mathrm{nm} \mathrm{GaAs}$ buffer layer. Based on this sample, single target QDs are deterministically integrated into circular mesa structures with a diameter of $400 \mathrm{~nm}$ (cf. inset of Fig. 1(b)). Here, the precise selection and central positioning of the target QD was achieved via in-situ electron-beam lithography using low-temperature cathodoluminescence spectroscopy. ${ }^{21,22}$ This fabrication technology allows us to realize high quality singlephoton emitters with excellent suppression of multi-photon emission events and narrow emission linewidth at a process yield close to $90 \%$. Such a high yield is in striking contrast to commonly used statistically random processing approaches. Details on sample structure, epitaxial growth, and in-situ electron-beam processing are reported in Ref. 14. Within our user-friendly approach, a twin-piston linear Stirling cryocooler with $6 \mathrm{~W}$ cooling power specified at $65 \mathrm{~K}$ and $23^{\circ} \mathrm{C}$ ambient temperature is used to cool the processed sample to cryogenic temperatures. Since the cryocooler is operated with almost no thermal load, a base temperature as low as $30 \mathrm{~K}$ is obtained. The cool-down begins instantaneously and takes about $30 \mathrm{~min}$, being as fast as LHe-flow cryostats in the same temperature regime. However, we would like to stress that in contrast to LHe-flow cryostats no limitations regarding the measurement time are present, making the Stirling cryocooler suitable for long-term measurements, e.g., at low signal-tonoise ratios. From an economic as well as ecological point of view, a striking advantage of Stirling cryocoolers is the low operational cost. This originates first from the expendability of LHe, and second from a low maximal power consumption of $280 \mathrm{~W}$, being significantly lower compared to closed-cycle pulse tube systems with a power consumption exceeding 1 kW. ${ }^{19,23}$

Thus, we are confident that these advantages will pave the way for a variety of applications of QD based singlephoton sources in the near future. The cooling principle is based on a counter-clockwise run Stirling cycle: A closed volume of gaseous helium is enclosed in a system containing two oppositely phased pistons sharing one axis and an offphase displacer piston with a regenerator medium through which the gas can flow into and out of an expansion volume inside the cold finger. The circle consists of two isochoric and two isothermal processes leading to a caloric intake as the gas leaves the expansion volume and thus resulting in a cooling of the cold finger. To demonstrate the performance of the Stirling cryocooler, a long-term measurement of the cold finger's temperature is displayed in Fig. 1(c). A mean base temperature of $28.79 \mathrm{~K}$ is achieved with the cryocooler working at full power. A standard deviation of $0.07 \mathrm{~K}$ over the complete 42 $\mathrm{h}$ measurement period is extracted from the data, proving long-term temperature stability without the need for any user intervention. The achieved temperature stability is thereby 
in the same range typically observed for LHe-flow cryostats equipped with a proportional-integral-derivative (PID) temperature controller $(\Delta \mathrm{T} \sim \pm 0.05 \mathrm{~K})$. We would like to point out that the temperature stability reported above facilitates also the operation of single-photon sources based on microcavities. ${ }^{24}$ In such cases, a temperature related spectral shift of the emitter and the associated detuning with respect to the cavity mode would only result in a significant drop of emission intensity for a temperature jitter exceeding about $1 \mathrm{~K}$, i.e., for temperature variations much larger than observed for our Stirling cryocooler. Since mechanical movement exports vibration to the surrounding, the cryocooler is placed on frequency-tuned, passive damping elements. The decoupled cooler, whose induced force in steady state operation is specified by the manufacturer to be about $0.5 \mathrm{~N} \mathrm{rms}$ along both relevant axes, shows vibrations with a base frequency of 46 $\mathrm{Hz}$ and amplitudes of about $1 \mu \mathrm{m}$ at the top of the cold finger, where the sample is mounted. The vibration amplitude was determined via white-light imaging and by comparing the maximal displacement of a structure on the sample surface to a well-known length scale. Since the detection spot-size in our $\mu \mathrm{PL}$ setup is $3 \mu \mathrm{m}$ in diameter, the remaining vibrations do not strongly affect the $\mu \mathrm{PL}$ signal of single QD structures, leading to similar count rates if compared to measurements using a standard LHe-flow cryostat ( $\mathrm{I}_{\text {Stirling }} / \mathrm{I}_{\mathrm{He}}$-flow $\approx 0.3$ ). We would like to mention that in both cases (He-flow cryostat and Stirling cryocooler), additional slow displacement drifts in the range of a few $\mu \mathrm{m}(<5 \mu \mathrm{m})$ occur on a time scale of $1 \mathrm{~h}$ and can be compensated by auto adjustment routines using piezo actuators. This issue can be solved by establishing a fibercoupling of the single-photon emission inside the cryocooler in the future. ${ }^{25}$ The LHe-flow cryostat used for reference measurements is placed on the same optical table and can be operated in parallel to the Stirling cryocooler without suffering from vibration export, meaning that there is no measurable reduction in the $\mu \mathrm{PL}$ signal in experiments using the LHe-flow cryostat while the Stirling cryocooler runs at full power. We would like to point out that this supports the applicability of table-top Stirling cryocoolers as almost vibration-free optical components in quantum optical experiments.

The optical properties of the sample are studied using a standard free-space $\mu \mathrm{PL}$ setup as depicted in Fig. 1(b). It is equipped with a diode pumped solid state laser $(\lambda=661 \mathrm{~nm})$, whose emission is focused by a microscope objective with a numerical aperture of 0.4 on the sample, placed either in the Stirling cryocooler or for reference in a LHe-flow cryostat. The PL signal is collected by the MO and detected by a spectrometer with a spectral resolution of $25 \mu \mathrm{eV}$. Autocorrelation measurements are performed with fiber coupled avalanche photo diodes in a HBT configuration as described elsewhere. ${ }^{22}$

\section{SAMPLE CHARACTERIZATION}

First, we study the optical properties of a single QD mesa structure mounted in the Stirling cryocooler at $35 \mathrm{~K}$ and at an excitation power of $0.4 \mu \mathrm{W}$. The corresponding $\mu \mathrm{PL}$ spectrum is depicted in Fig. 2(a). We observe a characteristic pattern of distinct and narrow excitonic emission lines originating from the single QD embedded in a deterministic mesa-structure. By analyzing polarization and power dependent measurements (cf. Fig. 2(a), inset and Fig. 2(b)), we identify exciton (X), biexciton (XX), and positively as well as negatively charged trion states $\left(\mathrm{X}^{+}, \mathrm{X}^{-}\right)$. Analyzing the polarization dependencies (cf. inset of Fig. 2(a)), a counter-phased behavior of the spectral positions of $\mathrm{X}$ and $\mathrm{XX}$ emission is observed, suggesting these lines to stem from exciton and biexciton of the same QD. ${ }^{26,27}$ Accordingly, a fine-structure splitting of the bright exciton's state of $(27 \pm 2) \mu \mathrm{eV}$ is extracted. Emission lines marked by $\mathrm{X}^{+}$and $\mathrm{X}^{-}$are identified as the corresponding charged states due to their relative spectral positions ${ }^{28}$ and due to the lack of any polarization dependence. This assignment is further supported by a more pronounced spectral jitter in the negatively charged trion's energetic position, resulting from a smaller effective mass of electrons compared to those of holes, therefore leading to a stronger quantum confined
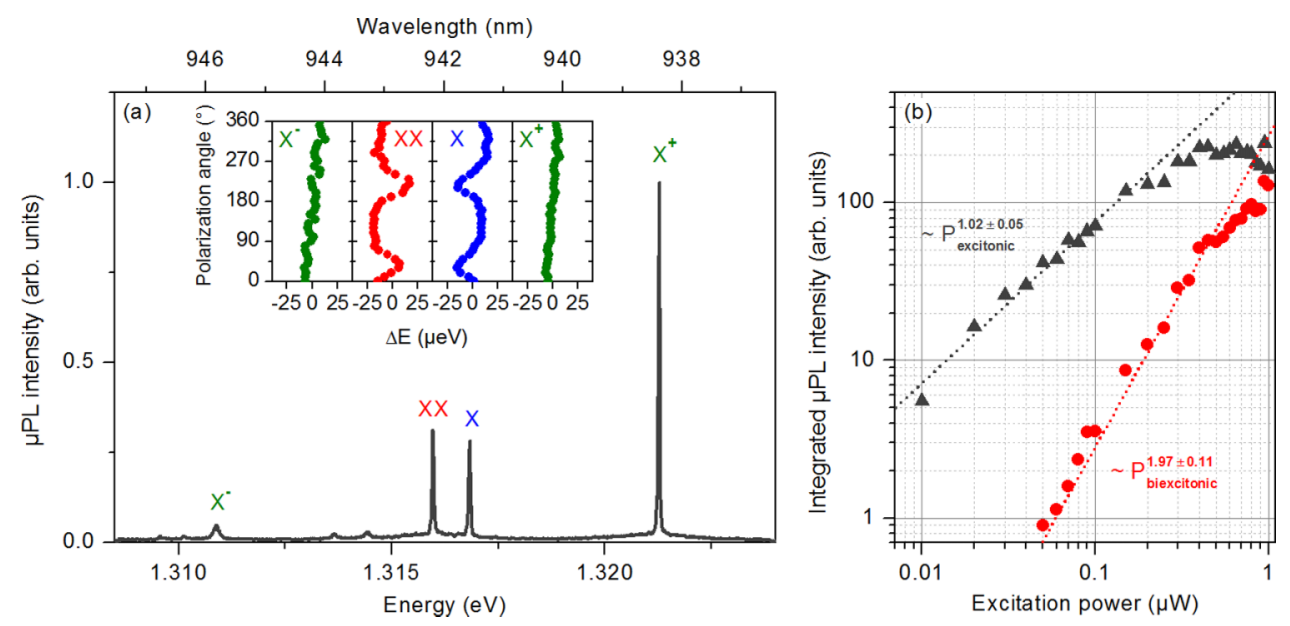

FIG. 2. Micro-photoluminescence ( $\mu \mathrm{PL}$ ) spectroscopy performed on a single InGaAs QD using a Stirling cryocooler. (a) High-resolution $\mu$ PL spectrum at $\mathrm{T}=35 \mathrm{~K}$. Spectrally narrow emission of exciton $(\mathrm{X})$, biexciton $(\mathrm{XX})$, and trion states $\left(\mathrm{X}^{-}, \mathrm{X}^{+}\right)$is observed. Inset: Analysis of polarization dependent $\mu \mathrm{PL}$ spectra yields a fine-structure splitting of the $\mathrm{X}$ state of $(27 \pm 2) \mu \mathrm{eV}$. (b) Integrated $\mu \mathrm{PL}$ intensity of the excitonic $\left(\mathrm{X}, \mathrm{X}^{-}\right.$, and $\left.\mathrm{X}^{+}\right)($triangles) and the biexcitonic (dots) emission versus excitation power $\mathrm{P}$. 
Stark-effect due to random charge fluctuations in the QD's surrounding. ${ }^{29,30}$ The observation of a prominent $\mathrm{X}^{+}$is attributed to an intrinsic p-type background doping during growth ${ }^{22}$ and the associated positive charging of the QD. Assignment of the biexcitonic state is finally carried out by means of power dependent measurements (see Fig. 2(b)).

The double logarithmic slope of the cumulative integrated $\mu \mathrm{PL}$ intensities of $\mathrm{X}, \mathrm{X}^{+}$, and $\mathrm{X}^{-}$(black triangles in Fig. 2(b)) is $1.02 \pm 0.05$ indicating the excitonic character of the emission, whereas the slope of the intensity of $\mathrm{XX}$ (red dots) is 1.97 \pm 0.11 indicating its biexcitonic character. ${ }^{31}$

In order to identify a suitable working point for further quantum optical studies, temperature dependent measurements were performed on the same QD mounted inside the Stirling cryocooler. Fig. 3(a) depicts the corresponding $\mu \mathrm{PL}$ spectra for temperatures from $30 \mathrm{~K}$ to $70 \mathrm{~K}$, each normalized with respect to the $\mathrm{X}^{+}$peak intensity at $30 \mathrm{~K}$. As expected, the QD emission experiences a pronounced red shift with increasing temperature, due to a decrease of the semiconductor's band gap. Interestingly, no significant quenching of the integrated intensities is observed up to about $55 \mathrm{~K}$, and QD emission features are clearly traceable and separable up to temperatures above $80 \mathrm{~K}$, indicating that the AlGaAs diffusion barriers introduced in the utilized samples (cf. Sec. II) efficiently reduce charge carrier losses. Fig. 3(b) exemplarily displays the extracted integrated $\mu \mathrm{PL}$ intensity of the $\mathrm{X}^{+}$ emission in dependence on the temperature. This observation is accompanied with a distinct line broadening of the QD emission (see Fig. 3(c)) due to increased contributions of phonon scattering, ${ }^{14}$ whereas for temperatures below $40 \mathrm{~K}$, the measured linewidth reaches the resolution limit of the experimental setup $(25 \mu \mathrm{eV})$. These observations underline the high optical quality and temperature stability in terms of the integrated emission intensity of the utilized deterministic single-QD structures.
It is worth mentioning that in the temperature range from $30 \mathrm{~K}$ down to $4 \mathrm{~K}-$ not accessible under Stirling operation so far - the homogenous linewidth of the excitonic states in InGaAs QDs further decreases from about $20 \mu \mathrm{eV}$ down to a few $\mu \mathrm{eV} .{ }^{14}$ For most envisaged applications (e.g., quantum key distribution), the spectral broadening present at $30 \mathrm{~K}$ is of minor importance, and even the implementation of more advanced concepts in quantum communication, such as the quantum repeater, relying on the emission of entangled photon pairs, ${ }^{32}$ has been shown to be within reach at this slightly elevated temperature. ${ }^{33}$

\section{COMPARATIVE PHOTON AUTO-CORRELATION}

In order to prove the applicability of the Stirling cryocooler also for quantum optical experiments, we conducted comparative photon statistics measurements using the Stirling cryocooler and a standard LHe-flow cryostat as a reference. Figs. 4(a) and 4(b) show the resulting photon autocorrelation functions $g^{(2)}(\tau)$ measured on the positively charged trion state of a deterministic single QD mesa structure. Both measurements were performed at an excitation power close to saturation of the charged exciton line and at a temperature of $29 \mathrm{~K}$ and $30 \mathrm{~K}$ inside the Stirling cryocooler and the LHeflow cryostat, respectively. The corresponding $\mu \mathrm{PL}$ spectrum of the single QD operated inside the Stirling cryocooler is depicted in Fig. 4(c), where the identification of QD states was carried out as mentioned above. Arrows indicate the detection bandwidth $(\sim 70 \mu \mathrm{eV})$ for the accumulation of $\mathrm{g}^{(2)}(\tau)$. Both correlation measurements show strong anti-bunching of the $\mathrm{g}^{(2)}(\tau)$-function at zero time delay, indicating the non-classical character of the emitted light. The timing resolution limited $\mathrm{g}^{(2)}(0)$ values (solid green lines) are 0.22 and 0.21 for Stirling cooling and LHe cooling, respectively. Considering the timing resolution of the avalanche photo diodes, one obtains
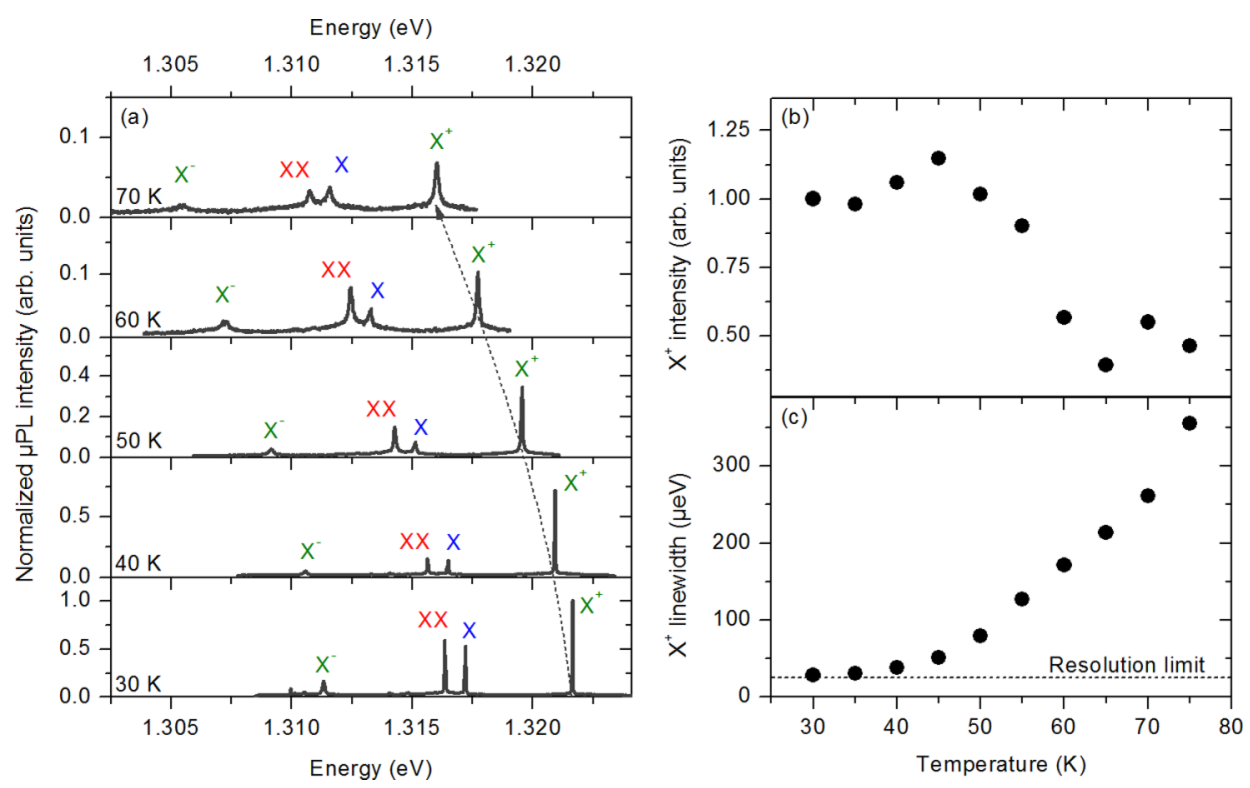

FIG. 3. (a) Temperature dependent $\mu$ PL spectra of the QD from Fig. 2 operated in the Stirling cryocooler. (b) and (c) Integrated $\mu$ PL intensity and linewidth (FWHM) of the positively charged exciton $\left(\mathrm{X}^{+}\right)$in dependence on the temperature. At low temperatures, the linewidth reaches the setup's spectral resolution limit $(25 \mu \mathrm{eV})$. 

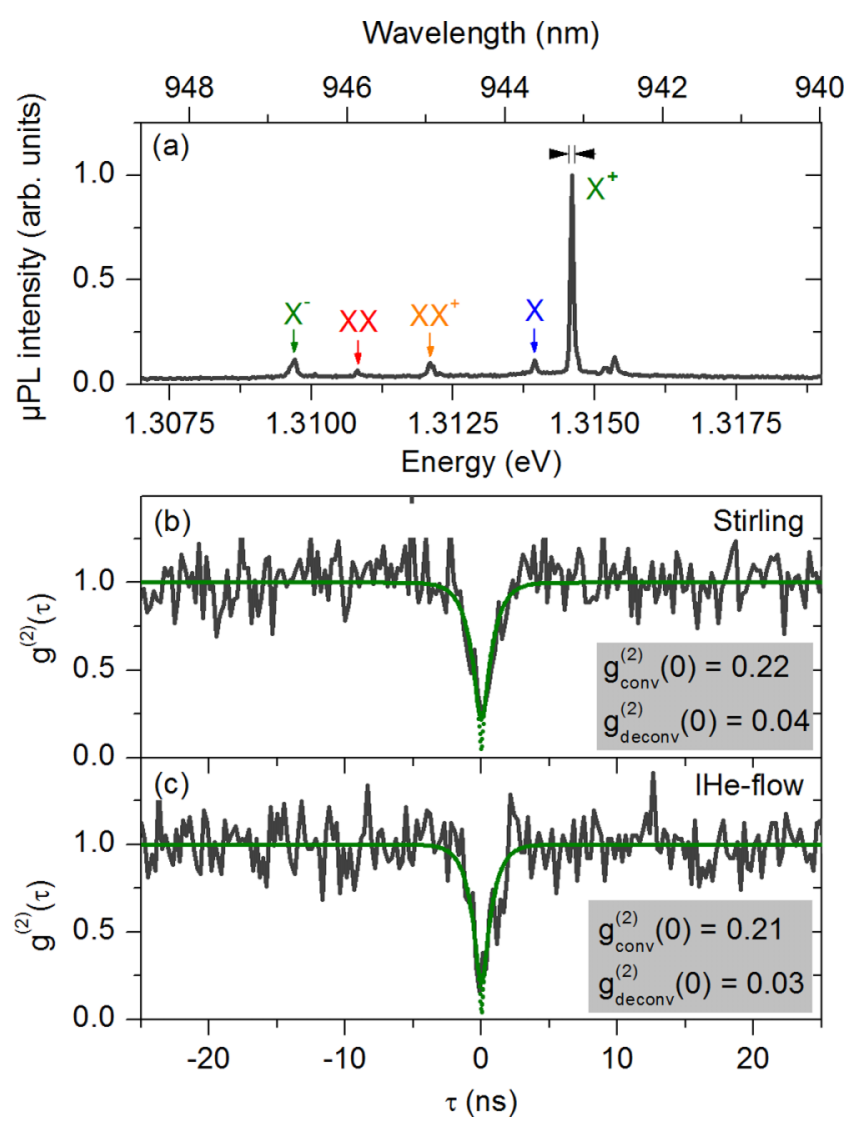

FIG. 4. (a) $\mu \mathrm{PL}$ spectrum of a single QD selected for measurements of the photon-autocorrelation function $\mathrm{g}^{(2)}(\tau)$. (b) and (c) demonstrate close to pure single-photon emission of the positively charged exciton $\left(\mathrm{X}^{+}\right)$while the sample is cooled via the Stirling cryocooler $(29 \mathrm{~K})$ and a LHe-flow cryostat $\left(30 \mathrm{~K}\right.$, as reference), respectively. The detector limited $\mathrm{g}^{(2)}(0)$-values (full lines) are 0.22 and 0.21 , and the deconvoluted values (dashed lines) are 0.04 and 0.03 .

$\mathrm{g}^{(2)}(0)$-values of $0.04(+0.12 /-0.04)$ and $0.03(+0.11 /-0.03)$ (dashed green lines).

\section{CONCLUSION}

In conclusion, we have demonstrated within a proof of principle experiment, the applicability of user-friendly Stirling cryocoolers to operate single-photon sources based on InGaAs QDs at low temperatures. The compact "off-the-shelf" Stirling cryocooler features low vibration levels (lateral displacement at the cold finger: $\sim 1 \mu \mathrm{m}$ ) and a base temperature below $30 \mathrm{~K}$. Implementing a Stirling cryocooler into a $\mu \mathrm{PL}$ setup, we performed optical studies on a deterministic single QD mesa structure. Utilizing this system, we carried out excitation power, polarization, and temperature dependent $\mu$ PL measurements on a single $\mathrm{QD}$, proving that Stirling cryocoolers are an attractive alternative to common LHe-flow cryostats or more expensive closed-cycle systems for temperatures $\gtrsim 30 \mathrm{~K}$. Moreover, we performed quantum optical studies showing single-photon emission with $\mathrm{g}^{(2)}(0)=0.04(+0.12 /-0.04)$ of a single-QD structure operated inside a Stirling cryocooler. The reported optical properties in combination with the possibility to perform $\mu \mathrm{PL}$ experiments on the same optical table prove the applicability of Stirling cryocoolers to provide a table-top single-photon source with high optical quality, suitable for applications in the fields of nanophotonics and quantum information technology. In the near future, our approach could be adapted to high efficiency state-of-the-art optically ${ }^{34,35}$ or electrically ${ }^{36}$ driven single-photon sources.

\section{ACKNOWLEDGMENTS}

This work was financially supported by the German Federal Ministry of Education and Research (BMBF) through the VIP-project QSOURCE (Grant No. 03V0630) and the German Science Foundation within the Collaborative Research Center 787 "Nanophotonic devices." We gratefully acknowledge expert sample preparation by R. Schmidt.

${ }^{1}$ P. Michler, A. Kiraz, C. Becher, W. V. Schoenfeld, P. M. Petroff, L. Zhang, E. Hu, and A. Imamoğlu, Science 290, 2282 (2000).

${ }^{2}$ A. J. Shields, Nat. Photonics 1, 215 (2007).

${ }^{3}$ M. D. Eisaman, J. Fan, A. Migdall, and S. V. Polyakov, Rev. Sci. Instrum. 82, 071101 (2011).

${ }^{4}$ S. Buckley, K. Rivoire, and J. Vučković, Rep. Prog. Phys. 75, 126503 (2012).

${ }^{5}$ G.-C. Shan, Z.-Q. Yin, C.-H. Shek, and W. Huang, Front. Phys. 9, 170 (2014).

${ }^{6}$ R. H. Hadfield, Nat. Photonics 3, 696 (2009).

${ }^{7}$ C. Kurtsiefer, S. Mayer, P. Zarda, and H. Weinfurter, Phys. Rev. Lett. 85, 290 (2000).

${ }^{8}$ M. Leifgen, T. Schröder, F. Gädeke, R. Riemann, V. Métillon, E. Neu, C. Hepp, C. Arend, C. Becher, K. Lauritsen, and O. Benson, New J. Phys. 16, 023021 (2014).

${ }^{9}$ S. Bounouar, M. Elouneg-Jamroz, M. Hertog, C. Morchutt, E. BelletAmalric, R. André, C. Bougerol, Y. Genuist, J.-Ph. Poizat, S. Tatarenko, and K. Kheng, Nano Lett. 12, 2977 (2012).

${ }^{10}$ M. J. Holmes, K. Choi, S. Kako, M. Arita, and Y. Arakawa, Nano Lett. 14, 982 (2014).

${ }^{11}$ S. Deshpande, A. Das, and P. Bhattacharya, Appl. Phys. Lett. 102, 161114 (2013).

${ }^{12}$ S. Deshpande, T. Frost, A. Hazari, and P. Bhattacharya, Appl. Phys. Lett. 105, 141109 (2014).

${ }^{13}$ P. Borri, W. Langbein, S. Schneider, U. Woggon, R. L. Sellin, D. Ouyang, and D. Bimberg, Phys. Rev. Lett. 87, 157401 (2001).

${ }^{14}$ M. Bayer and A. Forchel, Phys. Rev. B 65, 041308 (2002).

${ }^{15}$ P. Gold, A. Thoma, S. Maier, S. Reitzenstein, C. Schneider, S. Höfling, and M. Kamp, Phys. Rev. B 89, 035313 (2014).

${ }^{16}$ H. S. Nguyen, G. Sallen, C. Voisin, Ph. Roussignol, C. Diederichs, and G. Cassabois, Appl. Phys. Lett. 99, 261904 (2011).

${ }^{17}$ C. Matthiesen, A. N. Vamivakas, and M. Atatüre, Phys. Rev. Lett. 108, 093602 (2012).

${ }^{18}$ Y.-M. He, Y. He, Y.-J. Wei, D. Wu, M. Atatüre, C. Schneider, S. Höfling, M. Kamp, C.-Y. Lu, and J.-W. Pan, Nat. Nanotechnol. 8, 213 (2013).

${ }^{19}$ R. H. Hadfield, M. J. Stevens, S. S. Gruber, A. J. Miller, R. E. Schwall, R. P. Mirin, and S. W. Nam, Opt. Express 13, 10846 (2005).

${ }^{20}$ A. Veprik, S. Riabzev, G. Vilenchik, and N. Pundak, Cryogenics 45, 117 (2005).

${ }^{21}$ F. Donatini and L. S. Dang, Nanotechnology 21, 375303 (2010).

${ }^{22}$ M. Gschrey, F. Gericke, A. Schüßler, R. Schmidt, J.-H. Schulze, T. Heindel, S. Rodt, A. Strittmatter, and S. Reitzenstein, Appl. Phys. Lett. 102, 251113 (2013).

${ }^{23}$ M. Rau, T. Heindel, S. Unsleber, T. Braun, J. Fischer, S. Frick, S. Nauerth, C. Schneider, G. Vest, S. Reitzenstein, M. Kamp, A. Forchel, S. Höfling, and H. Weinfurter, New J. Phys. 16, 043003 (2014).

${ }^{24}$ J.-M. Gerard and B. Gayral, J. Lightwave Technol. 17, 2089 (1999).

${ }^{25}$ X. Xu, I. Toft, R. T. Phillips, J. Mar, K. Hammura, and D. A. Williams, Appl. Phys. Lett. 90, 061103 (2007).

${ }^{26}$ R. J. Young, R. M. Stevenson, A. J. Shields, P. Atkinson, K. Cooper, D. A Ritchie, K. M. Groom, A. I. Tartakovskii, and M. S. Skolnick, Phys. Rev. B 72, 113305 (2005).

${ }^{27}$ D. Sarkar, H. P. van der Meulen, J. M. Calleja, J. M. Becker, R. J. Haug, and K. Pierz, J. Appl. Phys. 100, 023109 (2006).

${ }^{28}$ S. Rodt, A. Schliwa, K. Pötschke, F. Guffarth, and D. Bimberg, Phys. Rev. B 71, 155325 (2005). 
${ }^{29}$ S. A. Empedocles and M. G. Bawendi, Science 278, 2114 (1997).

${ }^{30}$ V. Türck, S. Rodt, O. Stier, R. Heitz, R. Engelhardt, U. W. Pohl, D. Bimberg, and R. Steingrüber, Phys. Rev. B 61, 9944 (2000).

${ }^{31}$ E. Dekel, D. V. Regelman, D. Gershoni, E. Ehrenfreund, W. V. Schoenfeld, and P. M. Petroff, Phys. Rev. B 62, 11038 (2000).

${ }^{32}$ H.-J. Briegel, W. Dür, J. I. Cirac, and P. Zoller, Phys. Rev. Lett. 81, 5932 (1998).

${ }^{33}$ R. Hafenbrak, S. M. Ulrich, P. Michler, L. Wang, A. Rastelli, and O. G. Schmidt, New. J. Phys. 9, 315 (2007).
${ }^{34}$ J. Claudon, J. Bleuse, N. S. Malik, M. Bazin, P. Jaffrennou, N. Gregersen, C. Sauvan, P. Lalanne, and J.-M. Gérard, Nat. Photonics 4, 174 (2010), available online at http://www.nature.com/nphoton/journal/v4/n3/ abs/nphoton.2009.287.html.

${ }^{35}$ O. Gazzano, S.M. de Vasconcellos, C. Arnold, A. Nowak, E. Galopin, I. Sagnes, L. Lanco, A. Lemaître, and P. Senellart, Nat. Commun. 4, 1425 (2013).

${ }^{36}$ T. Heindel, C. Schneider, M. Lermer, S. H. Kwon, T. Braun, S. Reitzenstein, S. Höfling, M. Kamp, and A. Forchel, Appl. Phys. Lett. 96, 011107 (2010). 